\title{
El punto de vista atemporal en cosmología ${ }^{*}$ \\ (The Atemporal Viewpoint in Cosmology)
}

\author{
Olimpia LOMBARDI y Nicolás MOYANO LOZA
}

\begin{abstract}
Received: 05.05.2012
Final version: 01.07 .2012

BIBLID [0495-4548 (2013) 28: 78; pp. 499-516]

DOI: $10.1387 /$ theoria. 6154

RESUMEN: En su libro Time's Arrow and Archimedes' Point, Huw Price critica los argumentos de algunos cosmólogos contemporáneos acerca del origen cosmológico de la flecha del tiempo, quienes supuestamente no logran adoptar un punto de vista atemporal. En este artículo se analizan las críticas de Price a la propuesta de Stephen Hawking, argumentando que sus conclusiones se basan en supuestos cuestionables desde un punto de vista científico. Esta tarea abre el camino hacia un enfoque global no-entrópico de la flecha del tiempo, que permite establecer un nuevo diálogo con la propuesta de Price.

Palabras clave: flecha del tiempo; Price; Hawking; enfoque global no-entrópico.

ABSTRACT: In his Time's Arrow and Archimedes' Point, Huw Price criticizes the arguments of some contemporary cosmologists about the cosmological origin of the arrow of time, who supposedly fail to adopt an atemporal viewpoint. In this article we analyze Price's criticisms to Hawking's "no boundary" condition, by arguing that his conclusions rest on certain assumptions questionable from a scientific viewpoint. This task opens the way to a global and non-entropic approach to the arrow of time, which allows for a new dialogue with Price's proposal.

Keywords: arrow of time; Price; Hawking; global and non-entropic approach.
\end{abstract}

\section{Introducción}

Cuando, a fines del siglo XIX, Boltzmann desarrolló la versión probabilística de su teoría frente a las objeciones presentadas por Loschmidt y Zermelo, tuvo que enfrentar un nuevo desafío: ¿cómo explicar el altamente improbable estado actual de nuestro universo? Para responder esta pregunta, Boltzmann ofreció la primera explicación cosmológica de la asimetría temporal. Desde entonces, muchos autores han considerado que la flecha del tiempo tiene un origen cosmológico (por ejemplo, Davies 1974, Layzer 1975), entre ellos, Huw Price en su libro Time's Arrow and Archimedes' Point (1996). Pero la originalidad de su trabajo reside en su propuesta de comprender la asimetría temporal desde un punto de vista atemporal. Según Price, todo argumento en relación con la flecha del tiempo debe ser neutral respecto de la dirección del tiempo: dada la $t$-invariancia de las leyes fundamentales de la física, cualquier conclusión obtenida respecto de una dirección temporal también debe aplicarse a la dirección opuesta. El secreto para evitar errores es, entonces, abordar el problema desde un punto de vista atemporal, "arquimediano".

* El presente artículo ha sido realizado con el apoyo del CONICET (Subsidio PIP-597) y de la ANPCyT (Subsidio PICT-1432). 
En este artículo analizaremos las críticas de Price al modelo de universo "sin fronteras" de Stephen Hawking. Este análisis nos permitirá mostrar que, si bien la estrategia atemporal de Price es valiosa, sus conclusiones descansan sobre supuestos cuestionables desde un punto de vista científico. Aun cuando las tesis del autor han sido objetadas por diversos autores (Savitt 1996, Ridderbos 1997, Butterfield e Isham 1999, North 2002), aquí nos detendremos en ciertas dificultades que abrirán el camino hacia un enfoque global no-entrópico de la flecha del tiempo. Tal enfoque, que será presentado sobre el final del trabajo, permitirá establecer un nuevo diálogo con la propuesta de Price.

\section{Elpunto de vista atemporal de Price}

De acuerdo con Price, el error básico que se suele cometer al abordar el problema de la flecha del tiempo consiste en adoptar una perspectiva antropocéntrica que nos impide alcanzar una descripción objetiva de la asimetría temporal. Este error es muy común porque la diferencia entre pasado y futuro está tan profundamente enraizada en nuestra mente y nuestras percepciones que se nos hace muy difícil prescindir de ella. El modo de evitar cometerlo es, según Price, colocarse "fuera del tiempo", y considerar la realidad en términos atemporales. Este punto de vista arquimediano ("the view from nowhen") refleja la tendencia de la ciencia moderna en su adopción de perspectivas cada vez más descentradas de lo humano para comprender el mundo natural.

El punto de partida de Price es el problema de la asimetría temporal en los procesos termodinámicos (Capítulo 2) y en los fenómenos en los que participa la radiación (Capítulo 3). Tales análisis lo conducen a concluir que la respuesta al problema debe ser buscada en la cosmología (Capítulo 4). En este campo, comienza su argumentación señalando dos supuestos aceptados en la comunidad científica:

- La materia contenida en el universo temprano debe haber estado distribuida de un modo extremadamente suave y homogéneo ya que, si así no fuera, la gravedad habría tendido a agrupar la materia en cúmulos inmensos y las galaxias no se habrían podido formar.

- $\quad$ Si el universo es suficientemente denso, la fuerza atractiva de la gravedad será suficiente para superar la expansión presente, y el universo comenzará a contraerse hasta culminar en el llamado Big Crunch, un estado muy heterogéneo y grumoso en cuanto a la distribución de materia ${ }^{1}$.

Price considera que los científicos cometen aquí lo que denomina 'falacia del doble estándar temporal', que consiste en aplicar criterios diferentes a los dos extremos del universo. Desde un punto de vista atemporal, "nada en la física nos dice que un extremo del universo es objetivamente el inicio y el otro es objetivamente el fin" (Price 1996 , 84). Por lo tanto, cualquier argumento en favor de la "suavidad" del universo

\footnotetext{
${ }^{1}$ Desde el momento en el que Price escribió su libro, la hipótesis del Big Crunch fue reemplazada por la del Big Rip (Caldwell, Kamionkowski y Weinberg 2003), según la cual el universo finalmente se desintegrará en partículas subatómicas sin cohesión. No obstante, continuaremos la línea argumentativa de Price por su interés filosófico-conceptual.
} 
temprano debería valer como argumento acerca de la "suavidad" del universo tardío (Price continúa defendiendo esta posición en sus trabajos de 2002 y 2006). Según el autor, el argumento basado en la perspectiva atemporal también debe aplicarse al modelo inflacionario, que intenta explicar la homogeneidad del universo en sus etapas tempranas a partir de un período de expansión exponencial inicial que "suavizó" toda heterogeneidad inicial. Price sostiene que, dado que el modelo inflacionario explica la suavidad mediante un argumento estadístico, cae nuevamente en la falacia de doble estándar: si la expansión es vista en reversa, el mismo argumento debería aplicarse en el extremo del Big Crunch. Usualmente se señala que la "deflación" requeriría una conspiración extremadamente improbable de los fenómenos. Pero, para Price, el punto de vista atemporal revela que la transición desde el Big Bang ordinario a la fase inflacionaria implica el mismo tipo de conspiración improbable: "si la deflación es poco probable en un extremo, la inflación es poco probable en el otro" $(1996,86)$.

Price toma muy en serio el hecho de que las inferencias estadísticas sean ciegas a la dirección temporal: "a menos que ya haya una dirección temporal privilegiada, el razonamiento estadístico involucrado es tan bueno en una dirección como en la otra" $(1996,86)$. No obstante, como veremos, sus argumentos pierden su fuerza cuando se recuerda que las descripciones cosmológicas no se basan exclusivamente en consideraciones estadísticas.

\section{Las tesis de Hawking}

En la cosmología clásica hay sólo dos tipos de modelos de universo: o bien el universo existe desde un pasado distante infinito, o bien se inició con una singularidad. Hartle y Hawking (1983) encontraron que la aplicación de la gravedad cuántica a la cosmología abre una tercera alternativa: el espacio-tiempo puede tener extensión finita pero sin ninguna singularidad en su origen. Esto es lo que Hawking llama 'condición de ausencia de frontera' ('no boundary condition'): se trata de una condición de contorno nosingular que se aplica a la ecuación Wheeler-De Witt que rige el comportamiento de la llamada 'función de onda del universo'.

Pare comprender las críticas de Price no es indispensable considerar detalles técnicos (para ello, cf. Halliwell 1994, Ridderbos 2003). Sólo recordaremos que, al estudiar la métrica del espacio-tiempo que resulta de la aplicación de la condición de ausencia de frontera, Halliwell y Hawking (1985) encontraron que, cuando el radio del universo es pequeño, prima la homogeneidad e isotropía, pero a medida que se hace más grande, el universo se torna más irregular. Hawking explica esos resultados aplicados al modelo Big Bang-Big Crunch en su famoso trabajo "The arrow of time in cosmology" (1985), donde agrega que la ausencia de frontera implicaría homogeneidad e isotropía siempre que el universo fuese pequeño, es decir, no sólo en la etapa temprana de expansión, sino también en la etapa de contracción.

Sin embargo, poco después de que el artículo de 1985 fuera aceptado, discusiones con Raymond Laflamme y Don Page convencieron a Hawking de su error. En particular, Laflamme encontró un modelo más complejo en el que la fase de colapso del universo era muy diferente a la fase expansiva. Finalmente, Hawking, Laflamme y Lyons (1993) estudiaron un modelo de espacio-tiempo donde, partiendo de una situa- 
ción homogénea, la heterogeneidad aumenta durante toda la evolución, es decir, tanto durante la expansión como durante la contracción. Esos resultados llevaron a Hawking a rechazar sus conclusiones anteriores sobre la simetría del universo: "Este fue mi mayor error, o al menos mi mayor error en ciencia" $(1994,356)$.

Las tesis de Hawking acerca del origen de la asimetría temporal han sido objetadas sobre bases técnicas (cf., por ejemplo, Zeh 1994). Pero Price no se detiene en tales objeciones; desde su perspectiva, Hawking no logra enfrentar el problema de la flecha del tiempo desde una verdadera perspectiva atemporal, porque aplica la condición de ausencia de frontera sólo a un extremo del universo. En las próximas secciones analizaremos las críticas de Price, organizadas sobre la base de dos diferentes preguntas involucradas en el debate.

\section{Las preguntas "¿cómo es posible?” y "¿por qué es probable?”}

Respecto del problema de la dirección del tiempo, Steven Savitt (1996, 362) distingue entre dos cuestiones:

- La pregunta “ ¿cómo es posible?”: ¿cómo es posible formular un modelo de universo temporalmente asimétrico que satisfaga las leyes de la física invariantes bajo inversión temporal-o $t$-invariantes?

- La pregunta “¿por qué es probable?”: ¿qué razones tenemos para suponer que un universo temporalmente asimétrico es probable?

Si bien Price no distingue entre estas dos preguntas, implícitamente alude a ellas cuando exige que Hawking responda a ambas:

- “[Hawking] necesita explicar cómo se las ha arreglado para dar una conclusión asimétrica desde lo que manifiesta ser una teoría física simétrica respecto al tiempo" (1996, 87).

- "Hawking necesita un argumento más general, a los efectos de que los universos desorden-desorden sean imposibles (o, al menos, bastante improbables). Debe mostrar que casi todos los universos posibles tienen al menos un extremo temporal ordenado-o, equivalentemente, al menos un extremo desordenado" $(1996,89)$.

Price señala que hay una "escapatoria" ("loophole") a la aparente paradoja planteada por la pregunta "cómo es posible", la cual permite a una teoría física simétrica tener consecuencias asimétricas: "Esta es la escapatoria: una teoría física simétrica puede ser tal que todas o la mayor parte de sus posibles realizaciones sean individualmente asimétricas" $(1996,88)$. Price ilustra esta posibilidad con la familiar analogía de una fábrica que produce igual número de sacacorchos para zurdos y para diestros: la producción como un todo es simétrica, pero cada sacacorchos individual es espacialmente asimétrico. Como afirma Savitt, el punto puede ser formulado de un modo más preciso siguiendo la presentación de John Earman (1974): una teoría física $T$ es $t$-invariante si la inversión temporal $\mathbf{T}(m)$ de cada modelo $m$ de $T$ dinámicamente posible es también un modelo de $T$; la escapatoria consiste, entonces, en el hecho de que una teoría 
$T t$-invariante puede tener modelos $m$ que, en sí mismos, son temporalmente asimétricos.

Si Hawking hubiera proporcionado un modelo cosmológico asimétrico por medio de su condición de ausencia de frontera (como él mismo afirma luego de reconocer su error original), entonces habría dado una respuesta a la pregunta "cómo es posible". Pero Price considera que no lo ha logrado pues ha presupuesto la asimetría requerida, es decir, ha puesto la asimetría "con la mano". En otras palabras, Hawking no estaría en posición de explotar la escapatoria a causa de la ambigüedad referente al alcance de su condición de ausencia de frontera: "en efecto, la ambigüedad atrapa a Hawking entre dos errores: por un lado, asumir que el universo tiene un comienzo objetivo; por otro lado, no ver que, debido a que la aplicación de la condición de ausencia de frontera depende sólo del tamaño, ésta debe ser igualmente aplicable a los dos extremos temporales del universo" (Price 1996, 93). En la siguiente sección analizaremos los argumentos que llevan a Price a concluir que Hawking ha cometido esos dos errores respecto de la pregunta "cómo es posible", y en la Sección 6 consideraremos cómo la discusión experimenta un implícito viraje hacia la pregunta "por qué es probable".

\section{Los supuestos errores de Hawking}

\section{1 ¿Violación del punto de vista arquimediano?}

Comencemos por el segundo de los supuestos errores de Hawking: no toma en cuenta que la condición de ausencia de frontera debería aplicarse igualmente a los dos extremos temporales del universo, porque su aplicación depende sólo del tamaño del universo.

Price formula el problema de la flecha del tiempo en cosmología en términos del gradiente de entropía entre los dos extremos del universo. La razón para ello es que considera que es en la cosmología donde se encuentra la explicación de la asimetría temporal expresada por el segundo principio de la termodinámica. Pero, además, supone que la entropía depende sólo del tamaño del universo; por ello, imponer un estado de baja entropía al Big Bang y no al Big Crunch implica cometer una inadmisible falacia de doble estándar. De este modo, Price arriba a lo que considera el "dilema básico en cosmología": o bien aceptamos un universo estilo Gold, esto es, con baja entropía en ambos extremos, o bien nos vemos forzados a admitir que la flecha del tiempo es inexplicable mediante una física t-invariante (Price 1996, 82).

Una primera dificultad de esta posición es la que deriva de la definición de entropía involucrada en la discusión. Price transfiere con seguridad el concepto de entropía desde el campo de la termodinámica al de la cosmología. El problema es que la definición de entropía en cosmología es un tema muy conflictivo, incluso más que en termodinámica: no hay consenso entre los físicos acerca de cómo definir la entropía global del universo. En efecto, lo usual es trabajar exclusivamente con la entropía asociada a la materia y a la radiación, porque no se tiene una idea clara de cómo definir la entropía debida a la gravitación (cf. Earman 2006). Incluso dejando de lado estos inconvenientes, si la entropía del universo se considera una entropía fuera del equilibrio, se la puede definir de muchos modos posibles. Además, un eventual consenso no disiparía el pro- 
blema de encontrar la relación adecuada entre la entropía cosmológica y la entropía termodinámica tradicional (Mackey 1989).

Pero aun si pasáramos por alto este inconveniente y admitiéramos la definición de entropía gravitacional que ofrece Hawking, en este caso el incremento general de entropía en el universo se asocia con la tendencia general de los sistemas gravitacionales a evolucionar desde estados suaves y homogéneos hacia estados irregulares y heterogéneos. Los resultados de Hawking, Laflamme y Lyons (1993) muestran, precisamente, que la aplicación de la condición de ausencia de frontera permite una permanente evolución hacia la heterogeneidad, de modo tal que la entropía del universo aumenta durante la expansión y continúa incrementándose durante la contracción. Esto significa que, contrariamente al supuesto de Price, la entropia no depende sólo del tamaño del universo. Si Price estuviese en lo cierto, Hawking no sólo habría introducido la asimetría "con la mano", sino que también habría tenido éxito en concentrar la atención de la comunidad científica sobre un modelo inconsistente.

La idea de Price de que la entropía sólo depende del tamaño del universo se encuentra estrechamente relacionada con otra de sus tesis: la aplicación de las condiciones de contorno depende sólo del tamaño del universo. En efecto, ambas tesis son el resultado de suponer que, desde un punto de vista atemporal, si las leyes fundamentales son $t$-invariantes, no hay un modo objetivo de distinguir entre ambos extremos del universo más que por su tamaño-y, por lo tanto, por su entropía. Por lo tanto, en un universo Big Bang-Big Crunch, "no es un hecho objetivo cuál extremo del universo es el 'Bang' y cuál el 'Crunch"' (Price 1996, 84). Por este motivo Price insiste en que, si las condiciones de contorno se aplican a uno de los extremos, cuando el universo es pequeño, las mismas condiciones deben aplicarse al otro extremo, cuando el universo también es pequeño. A su vez, esto explica por qué Price presenta los universos orden-orden estilo Gold como una alternativa inevitable: si el único modo de distinguir entre ambos extremos del universo es por su tamaño, no es posible aceptar consistentemente un modelo Big Bang-Big Crunch con un gradiente de entropía entre sus extremos.

A su vez, la idea de que el tamaño es el único modo de distinguir entre ambos extremos del universo depende fuertemente de suponer que la cosmología sólo utiliza argumentos estadísticos para explicar la asimetría temporal (volveremos sobre este tema en la Sección 6). Price piensa exclusivamente en términos de la proporción de estados que, en cada extremo del universo, exhiben el grado de suavidad necesaria para explicar nuestras observaciones actuales. Por este motivo concluye que: "A pesar de su fuerza aparente, los argumentos estadísticos son sorprendentemente débiles. Rechazado el doble estándar, el hecho de que estos argumentos no funcionen hacia el pasado implica que no hay razones para esperar que funcionen hacia el futuro" $(1996,113)$.

Pero lo cierto es que Price no tiene razón: la cosmología no descansa exclusivamente sobre consideraciones estadísticas; sus explicaciones combinan argumentos estadísticos con descripciones dinámicas (una crítica análoga, pero en el contexto de la flecha termodinámica, puede hallarse en Ridderbos 1997). Como señala Craig Callender (1998), cuando los físicos postulan la inflación gravitacional o la teoría de cuerdas, están ofreciendo nuevas dinámicas, de acuerdo con las cuales-ellos esperan-el valor 
especial de las variables relevantes deje de ser especial. Esto vale también para la propuesta de la condición de ausencia de frontera: cuando Hawking estudia la evolución que resulta de la aplicación de dicha condición, está apelando a una descripción dinámica. Además, es interesante adelantar que, de este modo, Hawking obtiene un modelo del universo que no sólo es entrópicamente asimétrico-baja entropía-alta entropía-, sino también asimétrico en un sentido más fundamental, esto es, respecto de su propia estructura métrica, y ello permitiría una definición geométrica y, por tanto, noentrópica de la flecha del tiempo (volveremos a esta cuestión en la Sección 7, cuando presentemos el enfoque global no-entrópico). En conclusión, cuando se considera el modo en el que trabaja la cosmología actual, los razonamientos de Price pierden gran parte de su atractivo inicial.

\section{2 ¿Un comienzo objetivo del universo?}

Cuando Price examina los artículos de Hawking acerca de la condición de ausencia de frontera, encuentra que el autor describe el universo en un estado ordenado en el "tiempo inicial". Price considera que este uso de expresiones temporales pone de manifiesto que Hawking comete la falacia de doble estándar temporal: no logra adoptar el punto de vista arquimediano, ya que asume que un extremo del universo es "verdaderamente" el inicio. La pregunta es: ¿esas expresiones temporales son suficientes para sacar tal conclusión?

Cuando un físico usa $t$ como parámetro para describir la dinámica de su sistema—o el cosmólogo, para describir su modelo del universo-, comienza fijando un punto to y luego aplica las leyes dinámicas apropiadas. Usualmente, las ecuaciones dinámicas se resuelven para valores crecientes de $t$, con condiciones de contorno impuestas en $t_{0}=0$; en este caso, se las llama 'condiciones iniciales', y se dice que la evolución del sistema "comienza" en tales condiciones. Sin embargo, también se sabe que, si las ecuaciones dinámicas son $t$-invariantes, al resolverlas para valores decrecientes de $t$ se obtendría la evolución temporalmente especular, la cual es nomológicamente tan legítima como la original: la elección entre ambos "mellizos t-simétricos" es completamente convencional (cf. Castagnino y Lombardi 2009). Esto significa que el mero hecho de usar términos temporales en la descripción dinámica de un sistema no implica que la asimetría temporal haya sido introducida "con la mano". La pregunta relevante respecto de la flecha del tiempo es: ¿̇por qué elegir una determinada solución de las ecuaciones dinámicas y no la otra? La falacia de doble estándar consistiría en presuponer la validez de una de las dos soluciones desde una perspectiva subjetiva o antropocéntrica. Desde un punto de vista atemporal, el problema real es ofrecer un criterio $o b$ jetivo y no convencional para seleccionar una de las dos evoluciones nomológicamente posibles y temporalmente especulares, esto es, uno entre los dos mellizos $t$-simétricos.

Price parece no darse cuenta de que él mismo está brindando una respuesta a este problema en el contexto cosmológico cuando afirma que "un universo que colapsa sin deflación es un universo que se expande sin inflación. Es exactamente el mismo universo, bajo una descripción diferente pero igualmente válida" (1996, 86). La necesidad de elegir entre mellizos $t$-simétricos surge sólo si las dos soluciones describen evoluciones diferentes. Ahora bien, en el contexto cosmológico donde cada solución co- 
rresponde a un universo posible, ¿̇en qué sentido los dos universos posibles son diferentes? La respuesta obvia es: son diferentes porque están dispuestos en el tiempo en direcciones opuestas. Esta respuesta sería aceptable si estuviéramos intentando describir un sistema inmerso en un entorno que nos permitiera distinguir entre ambas soluciones; pero cuando el sistema es la totalidad del universo, no hay entorno. El enunciado 'dos universos posibles están dispuestos en el tiempo en direcciones opuestas' presupone la existencia de un tiempo único, "fuera" de ambos universos posibles y común a ambos, respecto del cual podemos decir que uno se opone al otro. Pero este supuesto es completamente contrario a la interpretación estándar de la relatividad general, según la cual el tiempo-o, mejor dicho, el espacio-tiempo-coexiste con el universo: no podemos distinguir entre el espacio-tiempo y el universo de la misma manera que distinguimos entre un recipiente y su contenido. De hecho, dos modelos matemáticos para el universo, definidos por $(M, g)$ y $\left(M^{\prime}, g^{\prime}\right)$, donde $M, M^{\prime}$ son las respectivas variedades diferenciales y $g, g^{\prime}$ son sus métricas, se consideran equivalentes si son isométricos, es decir, si existe un difeomorfismo $\theta: M \rightarrow M^{\prime}$ que convierte la métrica $g$ en la métrica $g^{\prime}$ (Hawking y Ellis 1973). En particular, los modelos relacionados por la transformación de inversión temporal $\mathbf{T}$ son isométricos; por lo tanto, dos modelos $m$ y $\mathbf{T}(m)$ se consideran descripciones equivalentes de la misma situación física (cf. también Reichenbach 1956 y Gold 1966 para una posición similar). Por lo tanto, las dos soluciones de las ecuaciones de campo de Einstein, una temporalmente especular respecto de la otra, son descripciones equivalentes de uno y el mismo universo (para una discusión pormenorizada de esta cuestión, cf. Castagnino, Lombardi y Lara 2003).

Una vez que se acepta esta conclusión, en cosmología no es necesario elegir entre dos mellizos $t$-simétricos. La flecha del tiempo es fijada por la estructura del universo como un todo o, en palabras de Price, por el modo "en el cual el contenido del universo bloque parece estar dispuesto asimétricamente respecto al eje temporal" (1996, 17). Por lo tanto, la solución temporalmente asimétrica de Hawking puede definir la flecha del tiempo a pesar de haber sido construida sobre la base de una elección convencional del punto inicial de la evolución: el universo asimétrico así descripto habría sido el mismo si se hubiese elegido el otro extremo del universo como condición inicial.

\section{6. ¿Qué tan probable es la asimetría temporal?}

Como se ha indicado, las críticas de Price son insuficientes para mostrar que Hawking no consigue aprovechar la "escapatoria lógica". Dejando de lado posibles objeciones técnicas, con su modelo sin frontera Hawking cumple con la exigencia de brindar una respuesta a la pregunta "cómo es posible". Sin embargo, tal exigencia original se identifica de pronto con otra completamente diferente: Hawking "debe mostrar que casi todos los universos posibles tienen al menos un extremo temporal ordenado" (1996, 89). Esto significa que, sin aclaración previa, se pasa de la pregunta "cómo es posible" a la pregunta "por qué es probable". La "escapatoria lógica" sufre una mutación análoga: después de ser presentada como "una importante escapatoria que permita a una teoría física simétrica tener consecuencias asimétricas" (1996, 88), Price afirma que, para sacar provecho de ella, Hawking tendría que mostrar "que la ausencia de 
frontera implica que todas (o casi todas) las historias posibles para el universo son asimétricas, con baja entropía (orden) en un extremo, y alta entropía (desorden) en el otro" $(1996,89)$.

Savitt (1996) está en lo correcto al afirmar que la pregunta "por qué es probable" no es la pregunta tradicional en las discusiones acerca de la flecha del tiempo. También es cierto que, para dar una respuesta completa a tal pregunta, se requieren argumentos científicos. No obstante, la cuestión puede ser considerada desde una perspectiva filosófica con el objetivo de analizar cuál sería una respuesta apropiada. Como Price no analiza los aspectos técnicos del complejo modelo de Hawking, nuestra estrategia será invertir la estrategia de Price. En lugar de averiguar si los universos ordendesorden son los más probables, preguntaremos: ¿los universos orden-orden estilo Gold son los más probables?²

El sencillo "modelo de las urnas" ("urn modep') de Ehrenfest es útil para presentar la respuesta a esta pregunta. El modelo consiste de dos cajas y un conjunto numerado de $n$ bolas distribuidas entre ellas. El sistema evoluciona de acuerdo con la siguiente regla temporalmente simétrica: en cada instante tomado a intervalos regulares, se selecciona un número aleatorio entre $1 \mathrm{y} n$, y la bola con ese número se transfiere de la caja que la contiene a la otra. La descripción de grano fino del sistema especifica su micro-estado: qué bola se encuentra en qué caja. Una descripción de grano grueso interesante es la que especifica, como macro-estado del sistema, únicamente el número total de bolas en cada caja, dejando de lado qué bola está en qué caja. Si elegimos una condición inicial macro en el instante $t_{i}$ en la cual todas las bolas están en una caja y seguimos la evolución, veremos que el sistema se aproxima al equilibrio-igual número de bolas en ambas cajas. Consideremos ahora la evolución de este modelo cuando una condición final macro, idéntica a la inicial, se impone en un tiempo posterior $t_{f}$. Para construir un conjunto de evoluciones consistentes con ambas macro-condiciones de contorno, debemos aceptar sólo aquellas evoluciones que parten en $t_{i}$ de la condición inicial macro- - todas las bolas en una caja—y alcanzan en $t_{f}$ la condición final macro-todas las bolas en la misma caja, de nuevo. Los cálculos sobre el modelo de Ehrenfest demuestran que sólo una pequeña proporción de todas las evoluciones es consistente con ambas macro-condiciones de contorno, y esta proporción decrece muy rápidamente cuando el número de bolas aumenta. Se han llevado a cabo cálculos análogos en sistemas clásicos y cuánticos, donde se calcula una cantidad $N$ relacionada con la probabilidad de simetría temporal (cf. Gell-Mann y Hartle 1994): en el caso clásico, $N^{-1}$ es la fracción de las trayectorias que cumplen tanto la condición inicial como la condición final; en el caso cuántico, $\mathrm{N}^{-1}$ es una medida de la probabilidad de la condición final, dada la inicial. En ambos casos $N^{-1}$ es muy pequeña para grandes sistemas con macro-

${ }^{2}$ En su reseña al libro de Price, Callender (1998) señala que puede haber razones empíricas para pensar que no vivimos en un universo estilo Gold: en un universo estilo Gold la radiación observable en el cielo nocturno debería ser mayor que la que vemos en la actualidad (cf. Davies y Twamley 1993). No discutiremos esta nueva versión de la paradoja de Olbers porque nuestro interés aquí no es si de hecho vivimos en un universo estilo Gold — tal situación podría ser el resultado de un azar extraordinario-, sino qué tan probable es un universo estilo Gold en el contexto de las teorías físicas contemporáneas. 
condiciones de contorno simétricas, y es de esperar que sea extraordinariamente pequeña para el caso del universo mismo. Vale la pena destacar que, en la construcción de este tipo de modelos, no se ha supuesto un comienzo objetivo del tiempo: dado que las ecuaciones dinámicas son $t$-invariantes, se obtendrían los mismos resultados si se las resolviese desde el instante final para valores decrecientes del tiempo.

Estas consideraciones ponen de manifiesto qué tan improbable es el caso de macro-condiciones de contorno temporalmente simétricas cuando son consideradas todas las evoluciones posibles (en la próxima sección mostraremos que la improbabilidad es aún mayor cuando se consideran las micro-condiciones de contorno). Puesto que los estados entrópicos en los extremos del universo son macro-condiciones de contorno, puede concluirse que los universos entrópicamente simétricos son extraordinariamente improbables en el marco de la legalidad que rige sus comportamientos.

Pero Price, atrincherado en su defensa de los universos estilo Gold, insiste en rechazar los argumentos que demuestran la baja probabilidad de los modelos entrópicamente simétricos. Cuando Davies señala el carácter extraordinariamente especial del micro-estado inicial necesario para obtener un macro-estado de baja entropía final, Price lo acusa de incurrir en la falacia del doble estándar, y agrega: "dejando de lado el doble estándar, la simetría gravitacional muestra que si el universo final es naturalmente grumoso, también lo es el universo inicial" $(1996,101)$. Esta cita pone claramente de manifiesto que, una vez más, Price olvida las consideraciones dinámicas y fundamenta su objeción en que la cosmología depende solamente de argumentos estadísticos; en particular, supone que las condiciones estadísticas pueden ser aplicadas independientemente a los dos extremos temporales del universo, sin ninguna restricción dinámica adicional. Pero el sencillo modelo de Ehrenfest muestra que, en la construcción de un modelo con macro-condiciones de contorno en ambos extremos temporales, tales condiciones no son la única restricción física: las evoluciones temporales también deben obedecer las leyes dinámicas del modelo. Este requisito es lo que descarta la mayor parte de las evoluciones compatibles con las dos macro-condiciones de contorno y, por tanto, explica la baja probabilidad de los modelos del universo estadísticamente simétricos.

Si bien, como hemos visto, tiene sentido preguntarse por la probabilidad de evoluciones que satisfagan ciertos macro-estados en dos tiempos diferentes, en física no es una estrategia legítima fijar el micro-estado del sistema en dos tiempos diferentes para computar una única evolución, porque esto equivale a sobredeterminar la solución de las ecuaciones dinámicas. Como señalan Gell-Mann y Hartle, "no podemos, por ejemplo, especificar ambas coordenadas y sus momentos conjugados tanto en el tiempo inicial como en el tiempo final. En general, las correspondientes soluciones de las ecuaciones clásicas del movimiento no existirían" (Gell-Mann y Hartle 1994, 335). Sin embargo, esto es precisamente lo que parece tener en mente Price al introducir dos modelos diferentes en que pueden establecerse las conexiones entre los productos de las dos condiciones de contorno. En el modelo que llama "de encuentro" ("meeting mo$\left.d e l^{\prime}\right)$, cualquier parcela de estructura u orden es un producto natural de las extremidades en ambas direcciones temporales. En el modelo que denomina "de mezcla" (" $m i$ xing model"), por el contrario, "la estructura u orden es normalmente un producto de 
una extremidad en una dirección, pero no de ambas" (Price 1996, 102-3). Price señala que Penrose y Hawking parecen suponer el modelo "de encuentro"; esto no es sorprendente, ya que el objetivo de la cosmología es obtener una descripción consistente del universo, no sólo desde un punto de vista estadístico sino también dinámico.

El modelo "de mezcla" sería el resultado de dos evoluciones diferentes para el mismo sistema: una calculada a partir de un extremo temporal y la otra calculada a partir del otro extremo. Con una actitud extremadamente benévola, Katinka Ridderbos (1997) sostiene que esta propuesta de Price exige una clarificación. Pero no parece haber clarificación alguna que evite una consecuencia totalmente inaceptable del modelo "de mezcla": en cada instante se asignarían al sistema dos estados diferentes, uno proveniente de la evolución iniciada en un extremo y el otro proveniente de la evolución iniciada en el otro extremo. La extraña idea de Price de un modelo "de mezcla" del universo parece ser el resultado de confundir la aplicación de dos condiciones de contorno estadísticas-macro—simétricas, una estrategia científicamente legítima, con la aplicación de dos condiciones de contorno dinámicas - micro—simétricas, lo cual resulta inaceptable desde el punto de vista de la práctica de la física.

Sin embargo, para continuar con el argumento, concedamos por un momento la posibilidad de un modelo "de mezcla" del universo, con el objetivo de explorar sus consecuencias. Supongamos, entonces, que es posible obtener la evolución del universo a partir de micro-condiciones de contorno aplicadas a sus dos extremos. Si, como parece exigir Price, a fin de no cometer la falacia del doble estándar temporal impusiéramos las mismas micro-condiciones de contorno dinámicas en ambos extremos de un universo regido por una cierta ley de evolución, entonces obtendríamos siempre una evolución temporalmente simétrica. En otras palabras, el enfoque temporalmente simétrico de Gold no sólo "debe ser considerada como la opción más plausible" (Price 1996, 112), sino que se convertiría en la única opción posible: el punto de vista atemporal haría de la simetría del universo una verdad a priori.

\section{El enfoque global no-entrópico de la flecha del tiempo}

El enfoque global entrópico de la flecha del tiempo, que encuentra sus raíces en la propuesta de Boltzmann, descansa sobre dos supuestos: que es posible definir la entropía para una sección completa del universo, y que hay un tiempo para el universo como un todo. Sin embargo, ambos supuestos presentan dificultades. En primer lugar, como ya señalamos, la definición de entropía en el marco de la cosmología es aún un tema muy controversial. En segundo lugar, cuando la relatividad general entra en juego, el tiempo se convierte en una dimensión de una estructura tetra-dimensional: ya no puede ser concebido como un parámetro de fondo, como en la física pre-relativista, que sirve para marcar la evolución del sistema. Por lo tanto, en cosmología el problema de la flecha del tiempo no puede ser legítimamente planteado, desde un principio, en términos del gradiente de entropía entre los dos extremos de un tiempo lineal y abierto.

Sin embargo, esas dificultades no son la principal razón para cuestionar el papel central que desempeña la entropía en el problema de la flecha del tiempo, sino que hay también un argumento conceptual para ello. La entropía es una propiedad fenomeno- 
lógica: un valor dado de entropía es compatible con diferentes configuraciones de un sistema. La pregunta es si existe alguna propiedad más fundamental que nos permita distinguir entre las dos direcciones del tiempo. Siguiendo la "herejía de la dirección del tiempo" de Earman (1974), es posible hablar del problema de la flecha del tiempo en términos de las propiedades geométricas del espacio-tiempo, independientemente de argumentos termodinámicos. Esta propuesta geométrica tiene prioridad conceptual respecto de la propuesta entrópica, ya que las propiedades geométricas del universo son más básicas que sus propiedades termodinámicas: la definición de entropía y el cálculo de la función de entropía para la totalidad del universo son posibles sólo si el espacio-tiempo tiene ciertas características geométricas definidas. Esta concepción global y no-entrópica de la flecha del tiempo fue desarrollada en una serie de trabajos recientes (Castagnino, Lara y Lombardi 2003a, 2003b, Castagnino, Lombardi y Lara 2003, Castagnino y Lombardi 2004, 2005, 2009, Aiello, Castagnino y Lombardi 2008) cuyos principales resultados se señalarán a continuación a fin de brindar un nuevo marco para evaluar la propuesta de Price.

Sobre el final de la Sección 5.1 señalamos que el modelo sin frontera de Hawking no sólo es entrópicamente asimétrico en el tiempo, sino que también es asimétrico en un sentido más fundamental, relacionado con su métrica. En efecto, para el cosmólogo el universo es un objeto espacio-temporal tetra-dimensional con propiedades geométricas; por lo tanto, puede ser simétrico o asimétrico a lo largo de la dimensión temporal. La cuestión es, entonces, averiguar si el universo es asimétrico a lo largo de la dimensión temporal: esta asimetría temporal permitiría distinguir entre ambas direcciones del tiempo y, así, definir una flecha del tiempo para el universo en su conjunto. Precisando aún más la idea: el universo es temporalmente asimétrico cuando sus propiedades geométricas son asimétricas a lo largo de la dimensión temporal. Pero puesto que las ecuaciones de campo de Einstein establecen la estrecha conexión entre la métrica del espacio-tiempo y la distribución de energía-materia, la tradicional pregunta acerca de si la flecha del tiempo es una propiedad del tiempo o de las cosas en el tiempo se disuelve: en el ámbito de la relatividad general, la asimetría refiere tanto a una propiedad del espacio-tiempo en sí mismo como a una propiedad de la distribución de la materia-energía en el tiempo.

No obstante, la respuesta al problema de la flecha del tiempo no es tan simple. Muchos espacio-tiempos diferentes, con topologías extremadamente variadas, son consistentes con las ecuaciones de campo, y algunos de ellos no permiten definir globalmente dos direcciones del tiempo, o incluso tampoco hablar de un tiempo único para el universo como un todo. Sólo es posible definir una flecha del tiempo para la totalidad del universo si el espacio-tiempo satisface ciertas condiciones geométricas (para detalles técnicos, remitimos a los artículos originales sobre el enfoque global noentrópico; cf. también Hawking y Ellis 1973):

a) Orientabilidad temporal. Esta característica permite que el conjunto de todos los semi-conos de luz del espacio-tiempo pueda dividirse en dos clases de equivalencia, $C+$ y $C$. Esto no se cumple en un espacio-tiempo cuyas secciones espaciales son análogos tridimensionales de una cinta de Moebius. Es claro que los nombres ' $C_{+}$' y ' $C$ ' son completamente convencionales, y pueden ser intercam- 
biados como deseemos: el único hecho relevante es que, para todos los semiconos, cada uno de ellos pertenece a una y sólo una de las dos clases de equivalencia.

b) Tiempo global. Sólo en ciertos modelos particulares de universo es posible sincronizar los relojes de todas las partículas del espacio-tiempo mediante un tiempo global, el cual tiene las características necesarias para desempeñar el papel del parámetro temporal de la evolución del universo. En términos geométricos, el espacio-tiempo es globalmente particionable en "instantes", es decir, en hipersuperficies tipo-espacio, cada una de las cuales contiene todos los sucesos simultáneos entre sí. Si, además, el intervalo de tiempo propio entre dos hipersuperficies de simultaneidad no depende de la trayectoria particular que se utilice para computarlo, entonces el tiempo global se convierte en tiempo cósmico.

Dadas estas condiciones, el problema puede ser formulado en términos tradicionales. Las ecuaciones de campo son $t$-invariantes en el sentido de producir mellizos $t$ simétricos: si $h_{i j}(\boldsymbol{x}, t)$ es una solución, $h_{i j}(\boldsymbol{x},-t)$ también es una solución, donde $h_{i j}$ es la 3 -métrica de cada hipersuperficie de simultaneidad y $t$ es el tiempo global. Pero, como nos enseña la "escapatoria" de Price, tal $t$-invariancia no impide describir un universo temporalmente asimétrico. Un espacio-tiempo es temporalmente simétrico o t-simétrico si es temporalmente orientable, admite un tiempo global $t$, y existe un tiempo global $t_{a}$ tal que $h_{i j}\left(\boldsymbol{x}, t_{a}+t\right)=b_{i j}\left(\boldsymbol{x}, t_{a}-t\right)$. Intuitivamente, la simetría temporal significa que, desde la hipersuperficie tipo-espacio correspondiente a $t_{\mathrm{a}}$, el espacio-tiempo "se ve igual" en ambas direcciones del tiempo. En definitiva, mediante las ecuaciones de campo $t$ invariantes puede describirse un universo $t$-asimétrico, tal que no exista ninguna hipersuperficie de simultaneidad que lo divida en dos "mitades", cada una especularmente simétrica respecto de la otra.

Sin embargo, la cosmología contemporánea no estudia todos los universos posibles compatibles con las leyes de la relatividad general, sino sólo el universo en el que vivimos: los hechos observacionales constriñen la aceptabilidad de los posibles modelos cosmológicos. Cuando se admite la expansión del universo y su homogeneidad e isotropía espacial a gran escala, los modelos son los conocidos como Friedmann-LemâitreRobertson-Walker (FLRW), que actualmente son estándar en cosmología y proporcionan la base para la teoría del Big-Bang. Tales modelos se describen en términos del tiempo cósmico $t$, y del factor de escala $a(t)$, usualmente conocido como "radio" del universo y que mide su "tamaño".

En los modelos FLRW, la asimetría temporal del espacio-tiempo puede manifestarse de dos maneras diferentes, de acuerdo a si el universo tiene puntos singulares en un extremo temporal ("Big Bang-Big Chill" o "Big Bang-Big Rip") o en ambos ("Big BangBig (runch"). Los modelos del primer tipo son manifiestamente $t$-asimétricos: puesto que $a(t)$ aumenta con $t$, no existe ninguna hipersuperficie de simultaneidad desde la cual el espacio-tiempo se vea igual en ambas direcciones temporales. En los modelos del segundo tipo, por el contrario, $a(t)$ tiene un valor máximo: por lo tanto, el espaciotiempo podría ser $t$-simétrico respecto del tiempo $t_{\mathrm{EM}}$ de expansión máxima de su radio, y esto es lo que sucede en un modelo de espacio-tiempo vacío. Sin embargo, 
puesto que se estudian universos que no están vacíos sino que incluyen campos de materia-energía, tales campos suelen romper la $t$-simetría, de modo tal que las dos mitades del espacio-tiempo respecto del tiempo de expansión máxima difieren en sus propiedades geométricas (cf. Halliwell 1994): $h_{i j}\left(\boldsymbol{x}, t_{\mathrm{EM}}+t\right) \neq h_{i j}\left(\boldsymbol{x}, t_{\mathrm{EM}}-t\right)$.

En definitiva, un universo Big Bang-Big Crunch puede ser un objeto $t$-asimétrico respecto de la métrica del espacio-tiempo, de un modo análogo en el que lo es el modelo sin frontera de Hawking. Esta asimetría geométrica, basada sólo en consideraciones físicas, permite distinguir ambas direcciones del tiempo cósmico, independientemente de cualquier consideración entrópica y desde una perspectiva completamente atemporal. En otras palabras, del mismo modo en que la diferencia entre los dos extremos de una flecha, digamos A y B, permite distinguir entre sus dos direcciones A-aB y B-a-A de un modo no meramente convencional ni dependiente de nuestra perspectiva antropocéntrica, la diferencia geométrica entre ambos extremos del universo permite definir una flecha del tiempo objetiva y purgada de toda expresión e intuición temporal.

\section{Un nuevo diálogo con la propuesta de Price}

Las diversas críticas señaladas en las secciones anteriores no deben opacar los aportes de Price al tradicional problema de la flecha del tiempo. El enfoque global noentrópico recoge explícitamente su enseñanza acerca de la necesidad de adoptar un punto de vista atemporal para la discusión del problema, y aprovecha la "escapatoria" que permite obtener evoluciones $t$-asimétricas a partir de leyes $t$-invariantes. A partir de su obra de 1996 ya no puede cometerse la falacia del doble estándar temporal ni pueden confundirse los conceptos de $t$-invariancia y $t$-simetría.

No obstante, al mismo tiempo el enfoque global no-entrópico pone al descubierto de un modo más evidente las limitaciones de los argumentos de Price respecto de la pregunta “¿cómo es posible?". Por un lado, el hecho de que las ecuaciones de campo de Einstein puedan tener soluciones $t$-asimétricas, y que efectivamente las tengan en el caso de los modelos Big Bang-Big Crunch de los que se ocupa Price, muestra que no es cierto que nada hay en la física que distinga entre ambos extremos del espaciotiempo ya que la única variable relevante es el tamaño del universo. Por otra parte, un enfoque que establece la diferencia entre las dos direcciones temporales en términos de la geometría del espacio-tiempo pone en evidencia la ceguera de Price a consideraciones dinámicas, que lo conduce a suponer que los únicos argumentos relevantes en cosmología son de naturaleza estadística.

Respecto de la cuestión de la probabilidad, puede formularse la pregunta: ¿qué tan probables son los universos geométricamente asimétricos a lo largo de la dimensión temporal? En el marco del enfoque global no-entrópico es posible probar, mediante un argumento sencillo, la probabilidad nula de la geometría temporalmente simétrica (cf. Castagnino, Lara y Lombardi 2003a, Earman 2004). En efecto, el espacio de las soluciones $t$-simétricas tiene una dimensión menor que la del espacio de todas las soluciones posibles. Esto significa que los universos de geometría temporalmente simétrica tienen una probabilidad nula respecto de la clase de referencia de todos los universos posibles compatibles con un modelo Big Bang-Big Crunch. Este resultado, ob- 
tenido sin violar el requerimiento arquimediano, desmiente la supuesta inevitabilidad de los universos estilo Gold: contrariamente a la opinión de Price, la simetría temporal es una característica muy específica que requiere un ajuste fino, abrumadoramente improbable, de todas las variables de estado del universo.

Si bien las críticas formuladas a la propuesta de Price limitan significativamente su aceptabilidad, no puede negarse que la entropía debe jugar algún papel en la discusión acerca de la flecha del tiempo. Es cierto que la diferencia geométrica entre los dos extremos de un universo $t$-simétrico permite establecer globalmente una diferencia no convencional ni antropocéntrica entre las dos direcciones del tiempo; pero también es cierto que es legítimo preguntarse por el modo en que la física da cuenta de la manifiesta direccionalidad temporal de los procesos locales que nos rodean (Price, comunicación personal). Precisamente a instancias de esta observación, el enfoque global noentrópico fue extrapolado desde el nivel global, del universo como un todo, al ámbito local, donde las teorías físicas describen evoluciones irreversibles que siempre proceden en una misma dirección del tiempo (Castagnino, Lara y Lombardi 2003b, Castagnino y Lombardi 2009). En efecto, si el universo cumple una condición muy general y ampliamente incorporada por los modelos cosmológicos actuales, la flecha del tiempo global se manifiesta localmente como un flujo cuatridimensional de energía que apunta en la misma dirección temporal en todos los puntos del espacio-tiempo. A su vez, este flujo de energía es lo que permite, a nivel local, romper con la simetría de los mellizos $t$-simétricos: los dos miembros del par, que resultan sólo convencionalmente diferentes desde el punto de vista de la teoría local t-invariante de la cual son soluciones, adquieren una diferencia sustancial cuando se los considera a la luz de la energía que transportan. De este modo se cuenta con un argumento físico para seleccionar como significativa la evolución cuya direccionalidad temporal coincide con la del flujo de energía y que, a su vez, manifiesta el comportamiento entrópico que predice el segundo principio de la termodinámica (cf. Aiello, Castagnino y Lombardi 2008).

Lamentablemente, Price no acusó recibo de estos trabajos desarrollados desde la perspectiva global no-entrópica, los cuales nunca fueron considerados en sus artículos posteriores. Incluso, en un trabajo muy reciente, Price (2011) parece haber renunciado a la defensa de los universos estilo Gold para volcarse al segundo cuerno del "dilema básico en cosmología" desde una perspectiva boltzmanniana: la flecha del tiempo no tendría una explicación objetiva. En ese trabajo, Price discute extensamente la "herejía de la dirección del tiempo" de Earman (1974), pero no menciona el enfoque global no-entrópico que se presenta explícitamente como un desarrollo de la propuesta herética original; de este modo, no toma en cuenta que muchos de los argumentos que esgrime contra Earman, e incluso contra otros autores como Horwich (1987) y Maudlin (2007), han recibido respuesta desde el enfoque global no-entrópico: sería interesante revisar los argumentos más recientes de Price desde el nuevo enfoque, pero esta tarea está más allá de los límites del presente trabajo.

\section{Conclusiones}

Como afirma Callender (1998) en su reseña, un gran libro es aquél que abre una discusión; esto es precisamente lo que ocurre con el libro de Price. Hemos enfocado nues- 
tros análisis críticos en el capítulo sobre cosmología, donde Price afirma que el debate contemporáneo acerca de la flecha del tiempo en cosmología está plagado de errores derivados de la falacia del doble estándar temporal. Sus argumentos son tan convincentes que, a primera vista, no parece posible rechazar sus conclusiones. Sin embargo, en este artículo hemos intentado poner de manifiesto que, en un análisis más detenido, los argumentos de Price pierden su atractivo original. La debilidad de sus críticas descansa principalmente en sobrevalorar el papel desempeñado por el razonamiento estadístico en cosmología: Price no toma en cuenta el hecho de que los modelos cosmológicos también deben obedecer leyes dinámicas, las cuales restringen las soluciones admisibles. Sobre esta base inadecuada construye la cadena de inferencias que lo conducen a la inevitabilidad de la simetría temporal.

El análisis de las críticas de Price al modelo sin frontera de Hawking también nos permite mostrar que la cosmología tiene sus propios recursos para distinguir entre ambas direcciones del tiempo sin recurrir a cuestiones entrópicas. Desde nuestro enfoque global no-entrópico, el problema de definir la flecha del tiempo debe ser encarado en términos de la t-asimetría respecto del tiempo global en un espacio-tiempo orientable.

No obstante nuestro desacuerdo, no puede negarse que Price realiza una importante contribución filosófica a la discusión de la flecha del tiempo con su propuesta de comprender la asimetría temporal desde el punto de vista atemporal. Después de leer su libro, nadie puede reflexionar acerca del problema sin intentar liberarse de su perspectiva temporal antropocéntrica, evitándose así los errores que resultan de proyectar nuestras intuiciones acerca de la diferencia entre pasado y futuro.

\section{REFERENCLAS}

Aiello, Matías, Mario Castagnino, y Olimpia Lombardi. 2008. The arrow of time: from universe timeasymmetry to local irreversible processes. Foundations of Physics 38: 257-292.

Butterfield, Jeremy, y Chris Isham. 1999. On the emergence of time in quantum gravity. En The Arguments of Time, ed. Jeremy Butterfield, 111-168. Oxford: Oxford University Press.

Caldwell, Robert, Marc Kamionkowski, y Nevin Weinberg. 2003. Phantom energy and cosmic Doomsday. Physical Review Letters 91: 071301.

Callender, Craig. 1998. The view from no-when. The British Journal for the Philosophy of Science 49: 135-159.

Castagnino, Mario, Luis Lara, y Olimpia Lombardi. 2003a. The cosmological origin of time-asymmetry. Classical and Quantum Gravity 20: 369-391.

Castagnino, Mario, Luis Lara, y Olimpia Lombardi. 2003b. The direction of time: From the global arrow to the local arrow. International Journal of Theoretical Physics 42: 2487-2504.

Castagnino, Mario, y Olimpia Lombardi. 2004. The generic nature of the global and non-entropic arrow of time and the dual role of the energy-momentum tensor. Journal of Physics $A$ (Mathematical and General) 37: 4445-4463.

Castagnino, Mario, y Olimpia Lombardi. 2005. A global and non entropic approach to the problem of the arrow of time. En Spacetime Physics Research Trends. Horizons in World Physics, ed. Albert Reimer, 73-108. New York: Nova Science.

Castagnino, Mario, y Olimpia Lombardi. 2009. The global non-entropic arrow of time: from global geometrical asymmetry to local energy flow. Synthese 169: 1-25.

Castagnino, Mario, Olimpia Lombardi, y Luis Lara. 2003. The global arrow of time as a geometrical property of the universe. Foundations of Physics 33: 877-912. 
Davies, Paul. 1974. The Physics of Time Asymmetry. Berkeley: University of California Press.

Davies, Paul, y Jason Twamley. 1993. Time-symmetric cosmology and the opacity of the future light cone. Classical and Quantum Gravity 10: 931-945.

Earman, John. 1974. An attempt to add a little direction to «The problem of the direction of time». Philosophy of Science 41: 15-47.

Earman, John. 2004. Curie's Principle and spontaneous symmetry breaking. International Studies in the Philosophy of Science 18: 173-198.

Earman, John. 2006. The «Past Hypothesis»: Not even false". Studies in History and Pbilosophy of Modern Physics 37: 399-430.

Gell-Mann, Murray, y James Hartle. 1994. Time symmetry and asymmetry in quantum mechanics and quantum cosmology. En Physical Origins of Time Asymmetry, ed. Jonathan Halliwell, Juan PérezMercader y Wojciech Zurek, 311-345. Cambridge: Cambridge University Press.

Gold, Thomas. 1966. Cosmic processes and the nature of time. En Mind and Cosmos, ed. Robert Colodny, Mind and Cosmos, 311-329. Pittsburgh: University of Pittsburgh Press.

Halliwell, Jonathan. 1994. Quantum cosmology and time asymmetry. En Physical Origins of Time Asymmetry, ed. Jonathan Halliwell, Juan Pérez-Mercader y Wojciech Zurek, 369-389. Cambridge: Cambridge University Press.

Halliwell, Jonathan, y Stephen Hawking. 1985. The origin of structure in the universe. Physical Review D 31: 1777-1791.

Halliwell, Jonathan, Juan Pérez-Mercader, y Wojciech Zurek, ed. 1994. Physical Origins of Time Asymmetry, Cambridge: Cambridge University Press.

Hartle, James, y Stephen Hawking. 1983. Wave function of the universe. Physical Review D 28: 2960-2975.

Hawking, Stephen. 1985. The arrow of time in cosmology. Physical Review D 32: 2489-2495.

Hawking, Stephen. 1994. The no boundary condition and the arrow of time. En Physical Origins of Time Asymmetry, ed. Jonathan Halliwell, Juan Pérez-Mercader y Wojciech Zurek, 346-368. Cambridge: Cambridge University Press.

Hawking, Stephen, y George Ellis. 1973. The Large Scale Structure of Space-Time. Cambridge: Cambridge University Press.

Hawking, Stephen, Raymond Laflamme, y Glenn Lyons. 1993. Origin of time asymmetry. Physical Review D 47: 5342-5356.

Horwich, Paul. 1987. Asymmetries in Time. Cambridge MA: MIT Press.

Layzer, David. 1975. The arrow of time. Scientific American 234: 56-69.

Mackey, Michael. 1989. The dynamic origin of increasing entropy. Reviews of Modern Physics 61: 981-1015.

Maudlin, Tim. 2007. The Metaphysics Within Physics. New York: Oxford University Press.

North, Jill. 2002. "What is the problem about the time asymmetry of thermodynamics?: A reply to Price. The British Journal for the Philosophy of Science 53: 121-136.

Price, Huw. 1996. Time's Arrow and Archimedes' Point: New Directions for the Physics of Time. Oxford: Oxford University Press.

Price, Huw. 2002. Boltzmann's time bomb. The British Journal for the Philosophy of Science 53: 83-119.

Price, Huw. 2006. The thermodynamic arrow: puzzles and pseudo puzzles. En Time and Matter, ed. Ikaros Bigi y Martin Faessler, 209-224. Singapore: World Scientific.

Price, Huw. 2011. The flow of time. En The Oxford Handbook of Philosopby of Time, ed. Craig Callender, 276-311. Oxford: Oxford University Press.

Reichenbach, Hans. 1956. The Direction of Time. Berkeley: University of California Press.

Ridderbos, Katinka. 1997. A point outside time?. Studies in History and Pbilosophy of Modern Physics 28: 523-535.

Ridderbos, Katinka. 2003. The thermodynamic arrow of time in quantum cosmology. En Philosophical Dimensions of Logic and Science, Synthese Library vol. 320, ed. Artur Rojszczak, Jacek Cachro y Gabriel Kurczewski, 179-194. Dordrecht: Kluwer Academic Publishers.

Savitt, Steven. 1996. The direction of time. The British Journal for the Philosophy of Science 47: 347-370. 
Zeh, Heinz-Dieter. 1994. Time (a-) symmetry in a recollapsing quantum universe. En Physical Origins of Time Asymmetry, ed. Jonathan Halliwell, Juan Pérez-Mercader y Wojciech Zurek, 390-404. Cambridge: Cambridge University Press.

Olimpia Lombardi es Ingeniera en Electrónica, Licenciada y Doctora en Filosofía por la Universidad de Buenos Aires (UBA). Investigadora del Consejo Nacional de Investigaciones Científicas y Técnicas (CONICET, Argentina), y Profesora de la Facultad de Ciencias Exactas y Naturales (UBA). Premio Konex 2006 en Lógica y Filosofía de la Ciencia. Se especializa en filosofía de la física y de la química. Ha publicado en Philosophy of Science, Foundations of Physics, Foundations of Chemistry, Studies in History and Philosophy of Modern Physics, International Studies in the Philosophy of Science, Synthese, entre otras.

Dirección: Instituto de Filosofía, Facultad de Filosofía y Letras, Universidad de Buenos Aires, Puán 480, Ciudad Autónoma de Buenos Aires, Argentina. E-mail: olimpiafilo@arnet.com.ar

Nicolás Moyano Loza es Licenciado en Filosofía por la Universidad Nacional de Mar del Plata (UNMP) Actualmente se encuentra realizando el doctorado de la Universidad Nacional de La Plata (UNLP) bajo la dirección de Olimpia Lombardi, y es becario del Consejo Nacional de Investigaciones Científicas y Técnicas (CONICET, Argentina). Ha publicado en revistas y participado en eventos académicos del ámbito nacional y regional.

Dirección: Departamento de Filosofía, Facultad de Humanidades, Universidad Nacional de Mar del Plata, Funes 3350, Mar del Plata, Argentina. E-mail: nicolasmoyanoloza@gmail.com 